\title{
Corrections to Group-Bayes Estimation of the Exponential Mean: A Preposterior Analysis
}

CONSTANCE van EEDEN and JAMES V. ZIDEK Department of Statistics, University of British Columbia 2021 West Mall, Vancouver BC, V6T 1Z2, Canada

\section{SUMMARY}

Corrections to "Group-Bayes estimation of the exponential mean: a preposterior analysis", Test 3 (1994), Num. 1, pp. 125-143.

\section{CORRECTIONS}

(i) The first inequality sign on the second line of (14) should be $<$.

(ii) The last inequality sign on line 6 of page 140 should be $>$.

(iii) The sentence starting on line 7 of page 141 should read:

For part (ii) note that

$$
\hat{\beta}_{\rho} \text { admissible in } \mathcal{C}^{\prime} \text { implies } \hat{\beta}_{\rho} \text { admissible in } \mathcal{C} \text {. }
$$

